\title{
Crosslinking effect on the deformation and fracture of monodisperse polystyrene-co-divinylbenzene particles
}

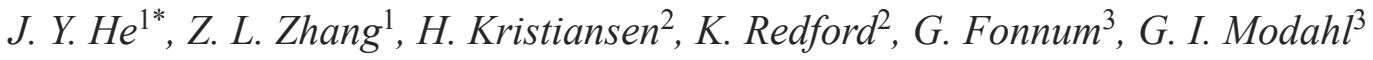 \\ ${ }^{1}$ NTNU Nanomechanical Lab, Department of Structural Engineering, Norwegian University of Science and Technology \\ (NTNU), 7491 Trondheim, Norway \\ ${ }^{2}$ Conpart AS, 2013 Kjeller, Norway \\ ${ }^{3}$ Invitrogen Dynal AS, P.O.Box 114, Smestad, 0309 Oslo, Norway
}

Received 27 September 2012; accepted in revised form 9 December 2012

\begin{abstract}
This study focuses on the effect of crosslinking density on the mechanical response of polystyrene-co-divinylbenzene (PS-DVB) particles under compression by means of nanoindentation-based flat punch method combined with SEM observation of particle morphologies. The monodisperse PS-DVB particles with about $5 \mu \mathrm{m}$ in diameter are produced by the Ugelstad activated swelling method and the crosslinking density defined as the weight percentage of activated crosslinker DVB during the preparation process varies from 2.0 to $55.3 \%$. Results show that the particle stress-strain behaviour is independent of the crosslinking density if the strain is less than $10 \%$. With increasing strain level over $10 \%$, a higher crosslinking leads to a stiffer behaviour of the particles. While slightly crosslinked (2.0 and $5.0 \mathrm{wt} \%)$ particles undergo plastic deformation with crazing and residual strain, highly crosslinked $(21.3,32.0$ and $55.3 \mathrm{wt} \%)$ counterparts experience perfectly viscoelastic deformation. The crosslinking density significantly influences the fracture property as well as the failure morphology. Slightly crosslinked particles become permanently deformed after compression, while highly crosslinked ones are entirely fragmented once a critical strain is reached.
\end{abstract}

Keywords: nanocomposites, nanoindentation, mechanical properties, crosslinking density, damage mechanism

\section{Introduction}

Micron-sized polymer particles have been widely used in chemical analysis and biotechnology, such as chromatographic supports, molecules carriers, additives in coating and ink products, and so on [13]. Recently increasing attention has been drawn to the use of polymer particles in electronic packaging technologies, for example Anisotropic Conductive Adhesive (ACA) by metallizing polymer particles $[4,5]$. The use of metalized polymer particles with a typical size in the range of $3 \sim 10 \mu \mathrm{m}$, as substitute for compact metal particles, brings significant advantages in terms of decreasing package size, reducing environmental impact, increasing the interconnection compliance and hence improving the electrical reliability. To obtain a low resistance connection, large contact area between the particles and the contact pads is preferred, and thus the particles are exposed to a deformation exceeding 30\% [6]. The mechanical characterization of particles under such a large deformation is necessary in order to ensure the electrical performance.

Polystyrene-co-divinylbenzene (PS-DVB) particles, due to their good mechanical properties, susceptibility to chemical modification and adaptability in a wide $\mathrm{pH}$ range, have been utilized in numerous applications, including in electronic packaging technology [7-10]. There are several methods available to synthesize crosslinked PS-DVB particles, such as suspension polymerization, dispersion poly-

\footnotetext{
${ }^{*}$ Corresponding author, e-mail: jianying.he@ntnu.no

(C) BME-PT
} 
merization, emulsion polymerization and seeded polymerization. However, these methods more or less have limitation on the preparation of PS-DVB particles for ACA. For example, by using suspension polymerization the particle size distribution is largely determined by stirring intensity, resulting in a broad size distribution and large particle diameter in the range of $20 \sim 1000 \mu \mathrm{m}$ [11]. Dispersion polymerization is usually utilized to prepare particles with a low crosslinking density less than $1 \mathrm{wt} \%$; otherwise it will lead to irregular shapes of particles [12]. The drawback of emulsion polymerization is that the technology for the required emulsion breaking is difficult to control [13]. Seeded polymerization is a relatively promising method to produce crosslinked particles but the enlargement of particles has been a hard work [14]. In contrast, the reformed seeded polymerization, known as Ugelstad method, has been developed to prepare highly monodisperse polymer particles which are wellcontrolled with a wide range of particle size, chemical composition and surface property $[15,16]$. In this study the Ugelstad method has been used to prepare monodisperse PS-DVB particles with same size and different crosslinking density.

Most theoretical and experimental studies on the mechanical properties of polymer materials have focused on bulky samples under tensile or shear test. The literature concerning mechanical properties of individual free-standing particles is relatively sparse and the existing ones mainly deal with particles larger than $100 \mu \mathrm{m}$ [17]. Due to the small volume, spherical geometry and large deformation of polymer particles involved in ACA application, the mechanical characterization of individual micron-sized particles challenges the conventional experimental techniques in aspect of testing scale and resolution [18]. To cope with this, a nanoindentation-based flat punch method has been developed to successfully measure mechanical response of individual particles under compression $[19,20]$. The deformation behaviour and failure properties of individual metal coated and uncoated polymer particles have been systematically investigated [2123]. In a previous study, a striking size effect on the stress-strain relationship of PS-DVB particles with identical crosslinking density but different size has been discovered, which indicates that the smaller the diameter is, the harder the particle behaves [24].

In this work, we extend our research to another horizon toward the effect of crosslinking density on the mechanical properties of PS-DVB particles. The particles with identical size but different crosslinking density are measured by using the nanoindentation-based flat punch method. The nominal compression stress-strain behaviors of individual particles are obtained, and the deformation process as well as fracture properties of particles are analyzed with respect to the crosslinking density. The results facilitate the design of the crosslinked PS-DVB particles with desired mechanical properties.

\section{Experimental}

\subsection{PS-DVB particles}

Five groups of PS-DVB particles were synthesized by means of Ugelstad method, which was based on seeded polymerization and emulsion polymerization, involving a two-step activated swelling process, as shown in Figure $1[15,16,25]$. The particle matrices were poly(styrene-co-divinylbenzene). The crosslinking density of particles was determined by the amount of crosslinker - activated DVB during the synthesis, covering the following percentages $2.0,5.0,21.3,32.0$ and $55.3 \%$ by weight. The size of particles was controlled around $5 \mu \mathrm{m}$. The size distribution within each batch, given by the coefficient of variance (C.V.) was less than $3 \%$, where C.V. was defined as the ratio of the standard deviation to the mean diameter. All PS-DVB particles were of amorphous type at room temperature. It was found that the glass transition temperature $T_{\mathrm{g}}$ of non-crosslinked linear polystyrene was about $100^{\circ} \mathrm{C}$, and $T_{\mathrm{g}}$ increased while the thermal expansion coefficient decreased with increasing crosslink-

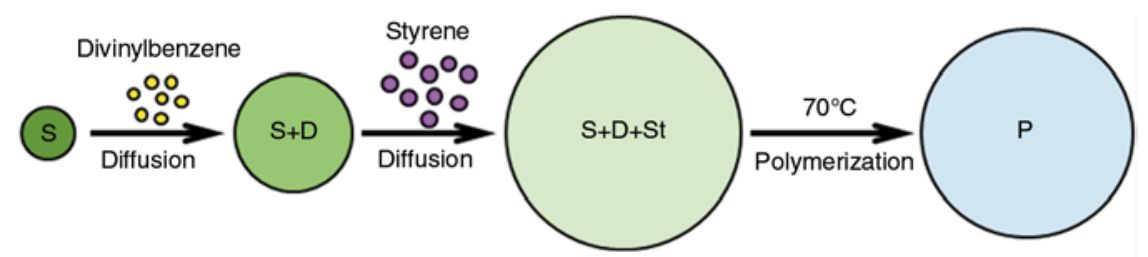

Figure 1. Preparation of highly monodisperse PS-DVB particles by using Ugelstad method, S: seed; D: divinylbenzene; St: styrene; P: particle 
Table 1. The properties of PS-DVB particles. The amount of DVB presents the crosslinking density of particles

\begin{tabular}{|l|c|c|c|c|}
\hline Particles & $\begin{array}{c}\text { DVB } \\
{[\mathbf{w t} \% \mathbf{]}}\end{array}$ & $\begin{array}{c}\text { Styrene } \\
{[\mathbf{w t} \% \mathbf{]}}\end{array}$ & $\begin{array}{c}\text { Diameter } \\
{[\boldsymbol{\mu} \mathbf{m}]}\end{array}$ & $\begin{array}{c}\mathbf{C V} \\
{[\mathbf{\%}]}\end{array}$ \\
\hline PSA & 2.0 & 98.0 & 5.1 & 0.8 \\
\hline PSB & 5.0 & 95.0 & 5.2 & 1.4 \\
\hline PSC & 21.3 & 78.7 & 5.3 & 2.8 \\
\hline PSD & 32.0 & 68.0 & 5.2 & 1.6 \\
\hline PSE & 55.3 & 44.7 & 5.2 & 1.6 \\
\hline
\end{tabular}

ing density [26, 27]. This implied that all particles remained amorphous during the measurement at room temperature. According to the crosslinking degree, particles could be divided into two groups in which PSA and PSB were slightly crosslinked and the rest three PSC, PSD and PSE were highly crosslinked. The dispersion method developed in the previous study was used to obtain individual particles [24]. The particles and the corresponding physical properties are given in Table 1.

\subsection{Nanomechanical test}

The mechanical testing of particles was performed by using the nanoindentation-based flat punch technique (TriboIndenter ${ }^{\circledR}$ Hysitron) [19]. It could be considered as an inverse nanoindentation schematically shown in Figure 2. Instead of an ordinary sharp indenter with pyramidal or spherical geometry for nanohardness measurement, a diamond flat punch with a flat end of about $10 \mu \mathrm{m}$ in diameter was designed to compress individual particles. The standard load-control mode for common nanoindentation measurement was employed, in which the applied load followed a predefined load function. A three-step loading protocol was used, including linear loading with $2 \mathrm{mN} / \mathrm{s}$ loading rate, $2 \mathrm{~s}$ holding at the peak load and linear unloading with $-2 \mathrm{mN} / \mathrm{s}$ loading rate. The applied force and the contact displacement were monitored during test and the real-

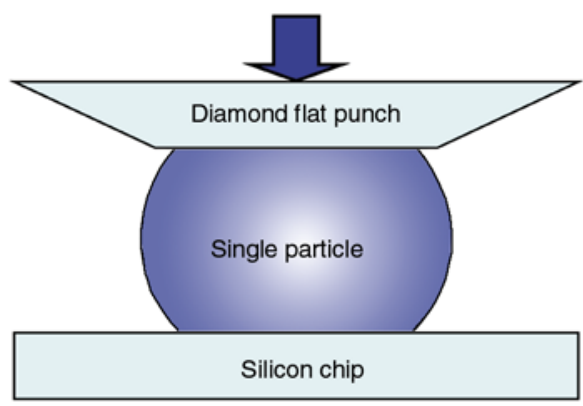

Figure 2. Schematic of the nanoindentation-based flat punch test time force-displacement relationship was obtained. The compression test was repeated on different individual particles from the same group to ensure the repeatability of the results. Experience with Ugelstad PS-DVB particles proved that particles from the same manufacturing batch showed remarkably consistent behaviour [24]. This indicated a homogeneous material from perspective of geometry and microstructure, even with highly reproducible experimental setup.

\subsection{SEM observation}

The as-prepared particle morphology was characterized by using scanning electron microscope (SEM) (FEI Helios NanoLab DualBeam ${ }^{\mathrm{TM}}$ ). The applied electron high tension (EHT) and the working distance (WD) were set to $5.0 \mathrm{kV}$ and $4.0 \mathrm{~mm}$, respectively. The fracture pattern was examined by a field emission SEM (Zeiss Ultra 55 LE FESEM $^{\circledR}$ ). Prior to imaging, the compressed particles rested in a clean environment for two weeks in order to complete the recovery of potential residual deformation. To minimize the damage from the electron beam and charge dissipation, the EHT and WD were set to relatively low values $0.5 \mathrm{kV}$ and $2.0 \mathrm{~mm}$.

\section{Results and discussion}

\subsection{Particles morphology}

The surface morphologies of five groups of PSDVB particles obtained by Ugelstad method are shown in Figure 3. The monodisperse size distribution and the spherical shape of particles are displayed, which indicates the well-controlled process of Ugelstad method. The particles with different crosslinking density keep nearly identical size about $5 \mu \mathrm{m}$ in diameter. While the surfaces of the slightly crosslinked particles are smoother than that of the highly crosslinked ones. Tiny pores on the surface of highly crosslinked particles exist and aggravate with the increasing crosslinking density. During the synthesis of particles, the polymerization is a coupled process of chemical (reaction) control and diffusion control. Resulting from an inhomogeneous distribution of crosslinking monomer, an inhomogeneous microstructure consisting of granules can form [28]. The granules can be considered as the aggregates of typically tiny particles with higher crosslinking density than the average. These highly crosslinked aggregates act as inclusions or second phase particles within polymer. 

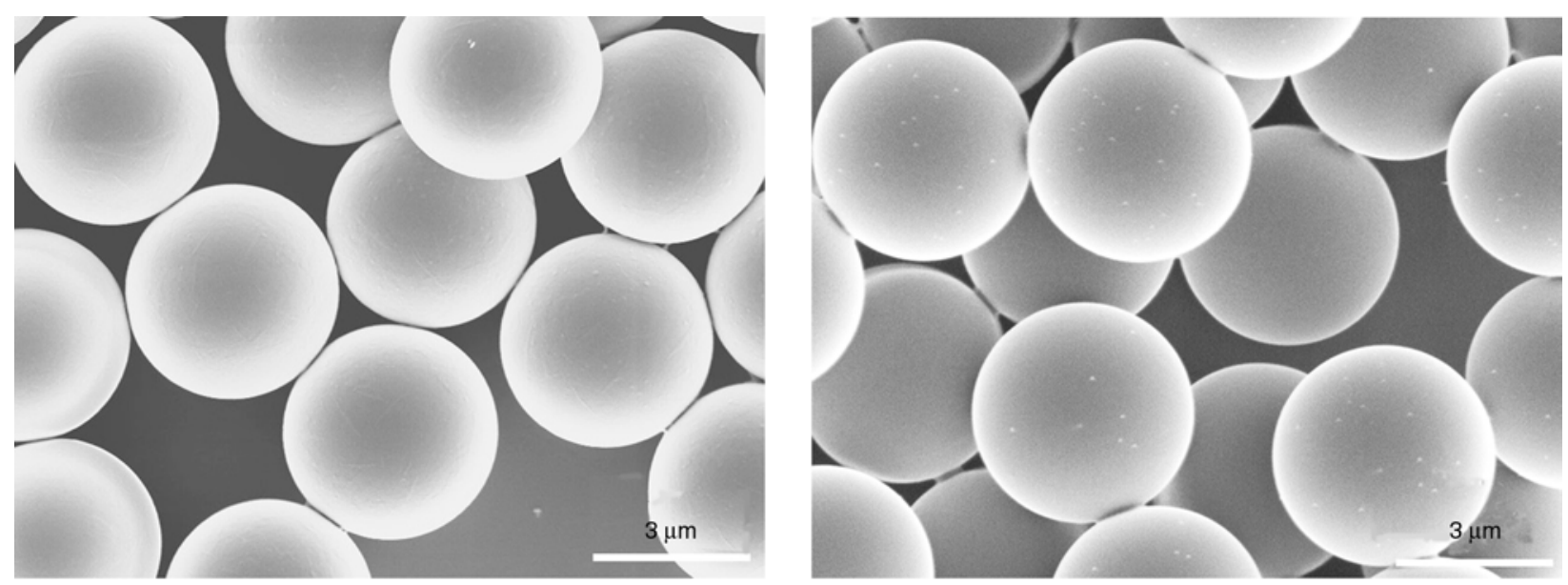

a)

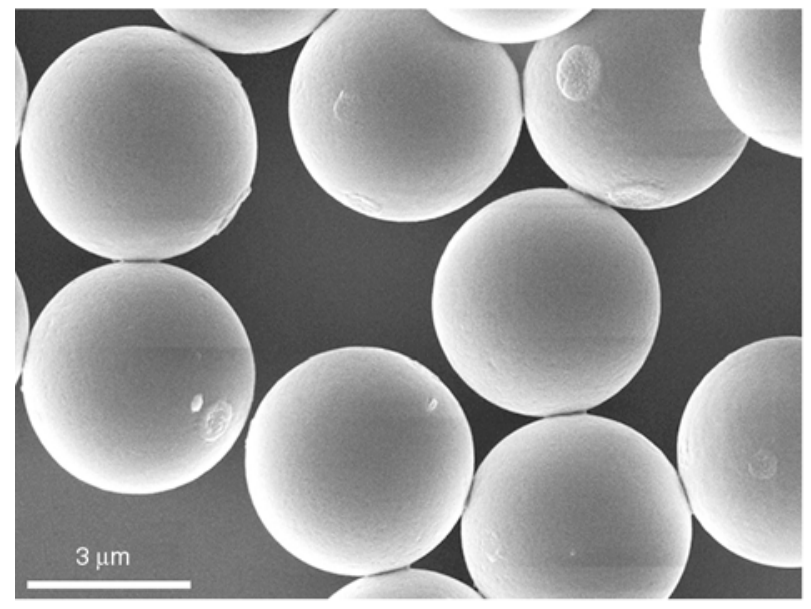

b)

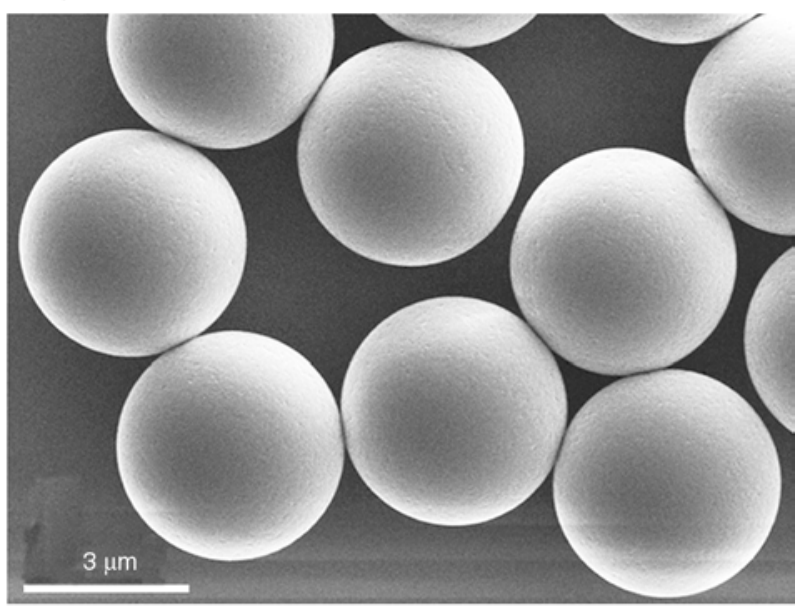

d)

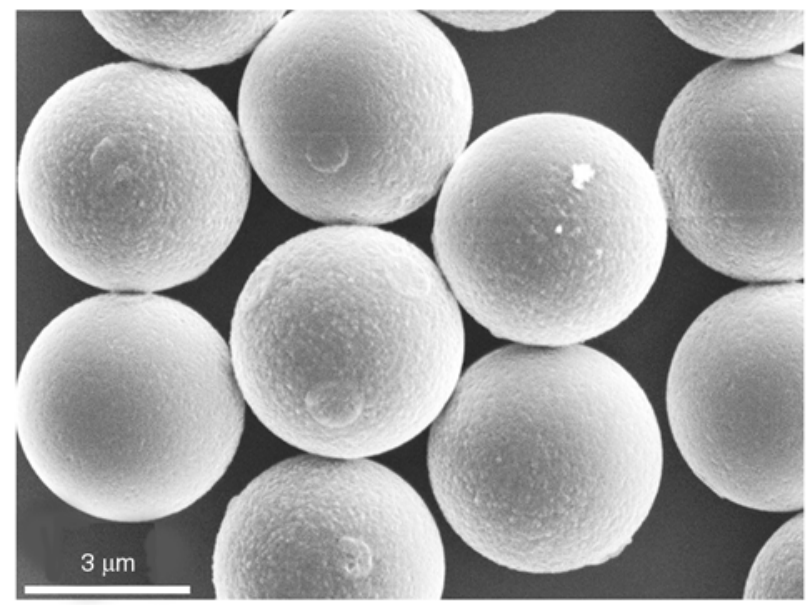

e)

Figure 3. SEM photograph of the PS-DVB particle. (a-e) present particle PSA, PSB, PSC, PSD and PSE, respectively. Electron high tension $(\mathrm{EHT})=5.0 \mathrm{kV}$; working distance $(\mathrm{WD})=4.0 \mathrm{~mm}$.

Although they are in an ultrafine scale, the presence of such aggregates strongly affects the mechanical properties of polymer matrix. It has been observed that the more densely crosslinked polymer is more heterogeneous and the crack in such polymer materials propagates in the regions with a relatively low crosslinking density between highly crosslinked clusters, which is consistent with our findings [28].

\subsection{Compression behaviour}

Systematic experiments show that the two slightly crosslinked particles have nearly identical behaviour and three highly crosslinked ones follow the same deformation pattern. The representative forcedisplacement curves of the lowest crosslinked particle PSA and the highest crosslinked PSE are plotted in Figure 4 and Figure 5, respectively. The com- 
pression peak loads are 1.5 and $20 \mathrm{mN}$ applied on PSA, and 5 and $20 \mathrm{mN}$ on PSE. In each figure two or three force-displacement curves of virgin particles are presented. It can be observed that the loading segments of the two groups of particles are repeatable and consistent. This is in contrast to bulky polymer materials in which the mechanical properties, especially the fracture point, often show a large scatter due to heterogeneous microstructure, and microscopic defects including variation in local crosslinking density and anisotropy, etc. [29]. The particle deformation is defined as the ratio of the contact displacement to the undeformed particle diameter. Under the compression at small peak load $1.5 \mathrm{mN}$, particle PSA hardly recovers any deformation, while PSE compressed to $5 \mathrm{mN}$ shows over $60 \%$ recovery of the deformation immediately after unloading. When compressed to the peak load $20 \mathrm{mN}$, the particle PSA deforms continuously although a significant displacement burst occurs on the loading segment of PSE. The displacement burst, also called 'pop-in', implies destructive failure of the particles [20]. Whereas the displacement increases a relatively large extent at the pop-in, the contact force drops because infinitesimal counterforce arises from the crushed particle. During unloading both particles have negligible deformation recovery. For the two slightly crosslinked particles we found that even increasing the peak load to $30 \mathrm{mN}$ there is no displacement burst. Moreover, the three highly crosslinked particles always show a large deformation recovery when unloading starts prior to the displacement burst.

The characteristic SEM images of particle PSA and PSE corresponding to the force-displacement curves in Figure 4 and Figure 5 are shown in Figure 6. All images are taken from the top view, in the direction of compression. While undeformed particle PSA has a smooth surface morphology, PSE is relatively rough. In Figure 6a, for particle PSA compressed at
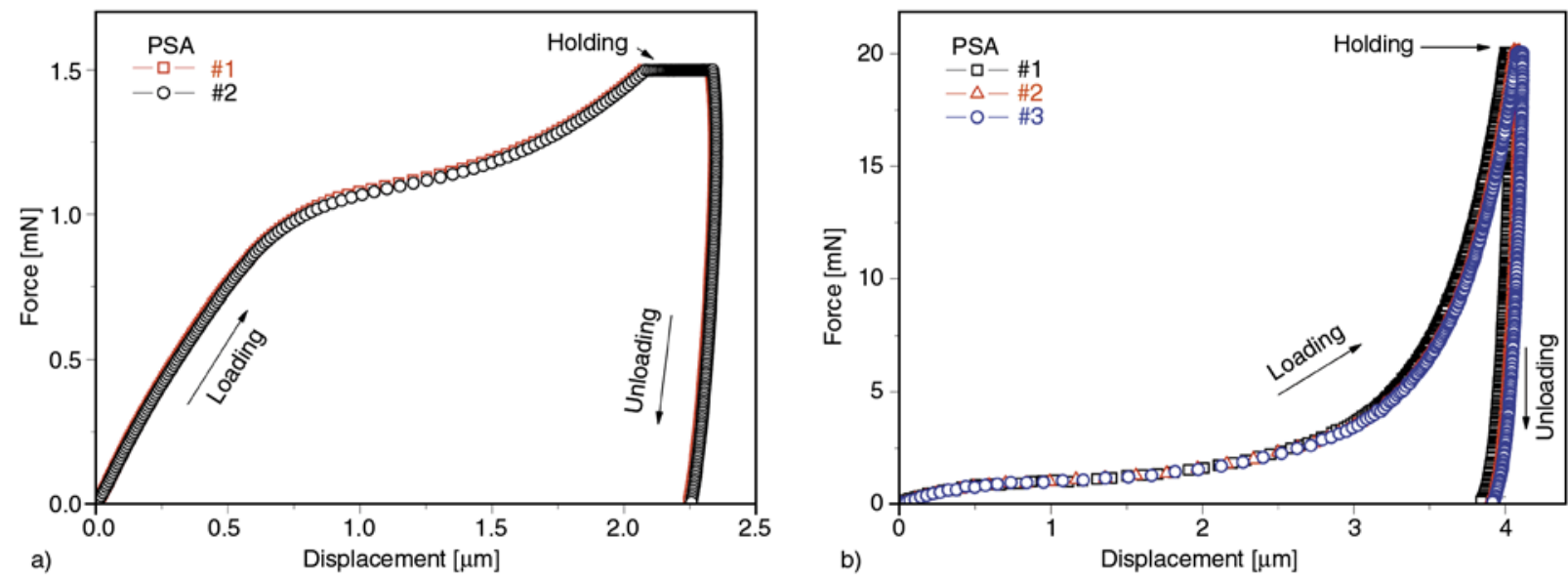

Figure 4. The representative force-displacement curves of slightly crosslinked particle PSA at different peak load (a) $1.5 \mathrm{mN}$ and (b) $20 \mathrm{mN}$

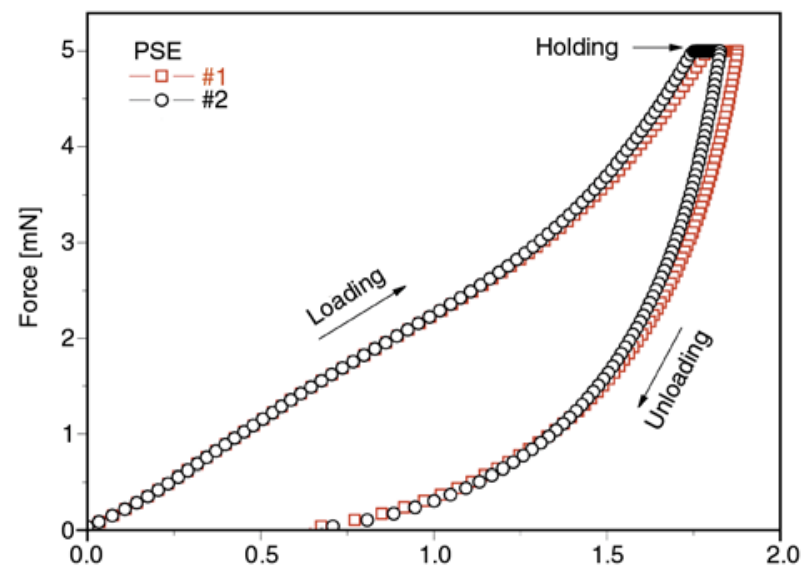

a)

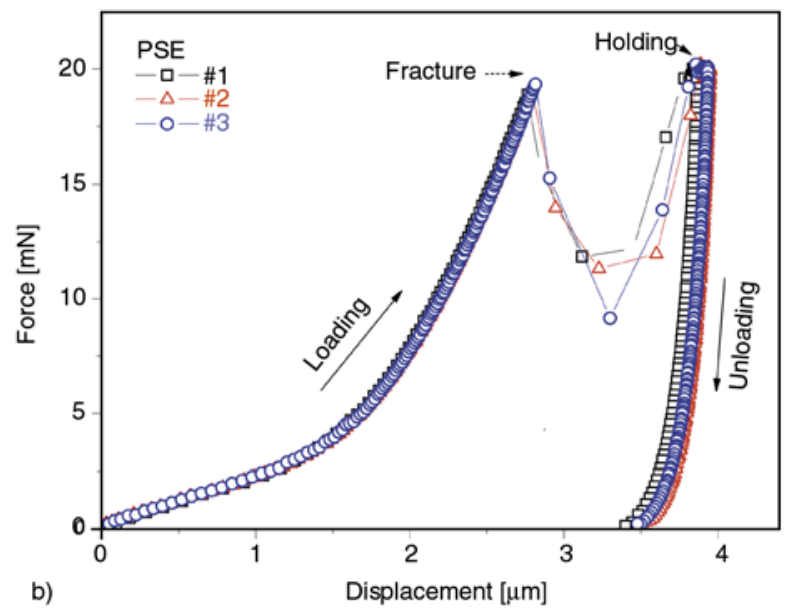

b)

Figure 5. The representative force-displacement curves of highly crosslinked particle PSE at different peak load (a) $5 \mathrm{mN}$ and (b) $20 \mathrm{mN}$ 

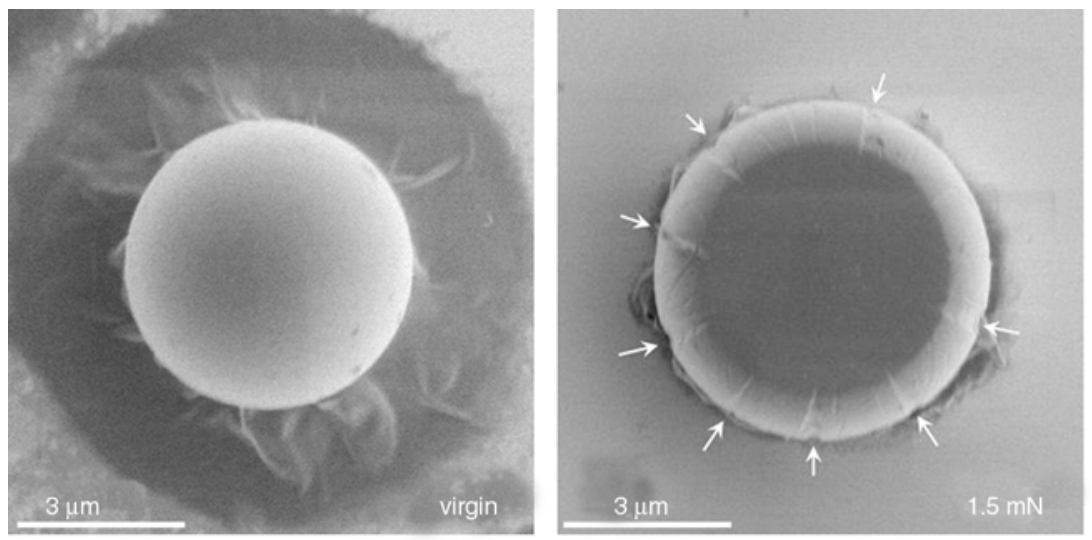

a)
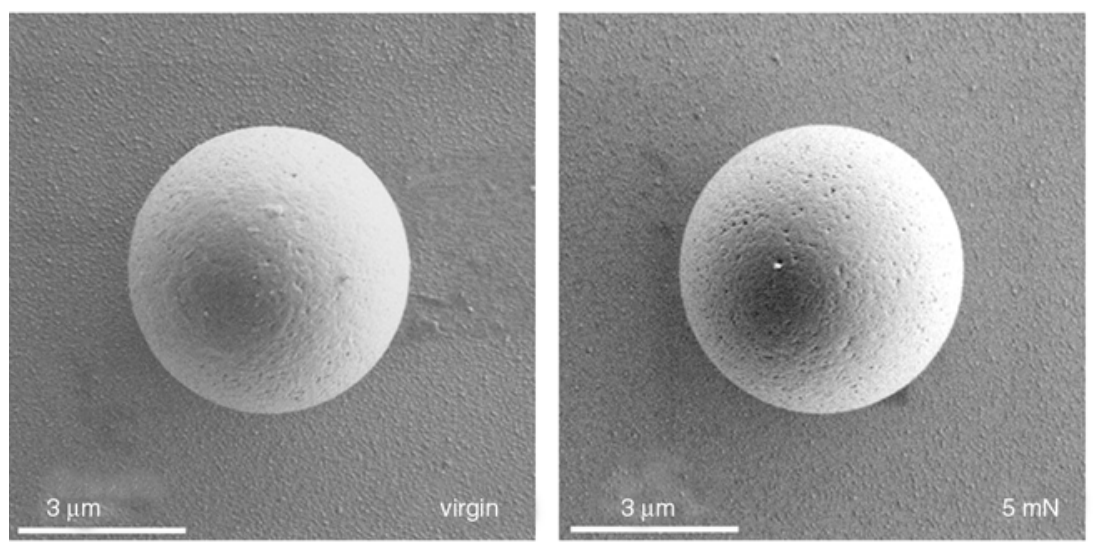

b)

Figure 6. The characteristic SEM images of particle morphology after compression, (a) PSA and (b) PSE. Electron high tension $(\mathrm{EHT})=0.5 \mathrm{kV}$; working distance $(\mathrm{WD})=2.0 \mathrm{~mm}$.

$1.5 \mathrm{mN}$ peak load, a flattened surface area is observed which corresponds to the expected contact area and indicates the residual deformation. Isolated crazes can be observed, as indicated by arrows in the corresponding micrograph. The craze formation is originated from the tensile region of deformed drum-like particles under compression. When the peak load is up to $20 \mathrm{mN}$, the particle becomes oblate with a large number of interacted crazes around the edge. In Figure 6b, particle PSE shows a drastically different behavior from particle PSA. In the case of compression at the peak load of $5 \mathrm{mN}$, there is no craze, even no evidence of the contact area observed. The particle completely recovers the residual deformation presented in Figure 5a and has identical size with the undeformed one. Further compressing the particle to $20 \mathrm{mN}$, fragmentation occurs.

The results of compression test and SEM observation suggest that the slightly crosslinked particles behave plastically with the presence of local brittle crazes and residual deformation after unloading; while the highly crosslinked counterparts display viscoelastic behaviour, which recover completely within a certain time after unloading.

\subsection{Deformation process and fracture}

Both microstructure and geometry influence the mechanical response of the particle. To eliminate the effect of variation in particle size, the force-displacement results are normalized to the stressstrain relationship. Similar to the previous study, the nominal compression stress-strain relationships of the particles are adopted, as shown by Equations (1) and (2) [18]:

$$
\begin{aligned}
\sigma_{\mathrm{C}} & =\frac{P}{\pi R^{2}} \\
\varepsilon_{\mathrm{C}} & =\frac{D}{2 R}
\end{aligned}
$$

where $\sigma_{\mathrm{C}}$ is the nominal compression stress, $\varepsilon_{\mathrm{C}}$ is the nominal compression strain, $R$ is the initial radius of the particle, $P$ is the contact force, and $D$ is the particle displacement. The nominal compression stress-strain curves of the five groups of parti- 

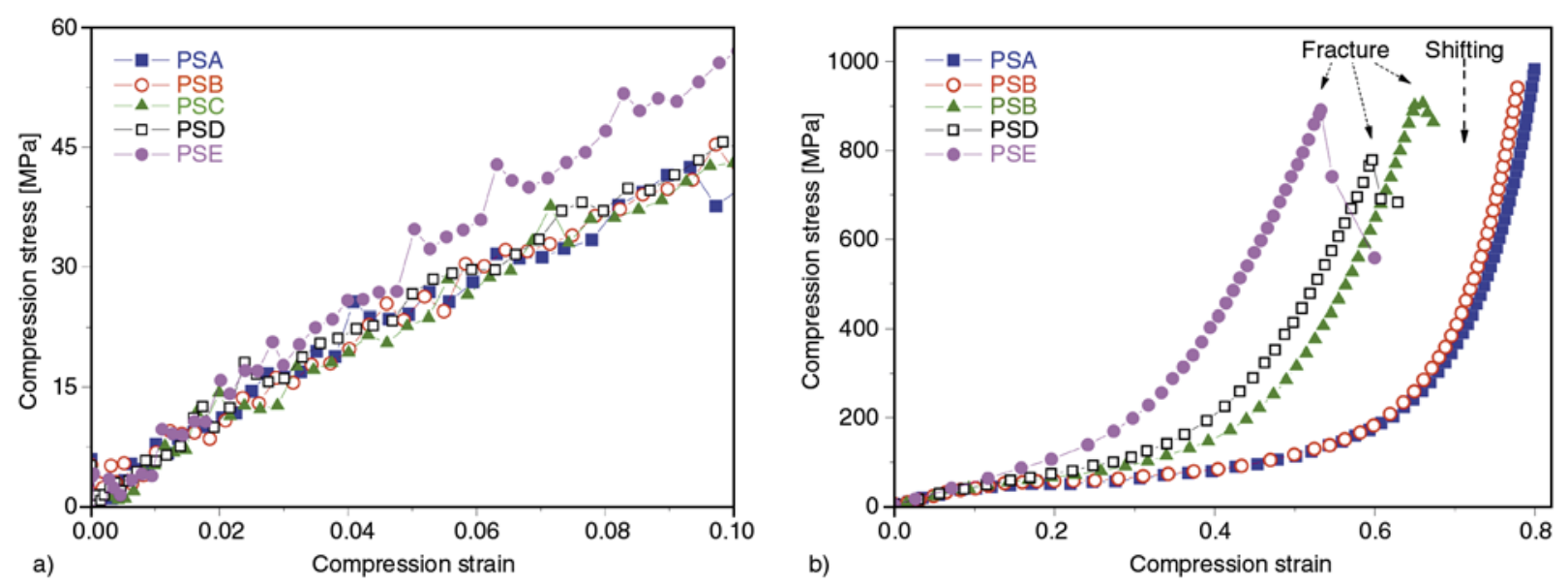

Figure 7. Compression stress-strain curves of five PS-DVB particles (a) at small strain level and (b) at large strain level

cles at different strain levels are plotted in Figure 7. The focus of this study is on the loading behaviour of particles, thus the holding and unloading segments on the stress-strain curves are disregarded. During the large deformation of particles under compression, the contact between the particle and punch/substrate changed from point to a finite area continuously and the spherical geometry induce highly nonlinear stress-strain behaviour.

At small strain level below 10\%, as shown in Figure $7 \mathrm{a}$, the stress-strain behaviour of particles appears independent on the crosslinking density, indicating that the reduced $E$-modulus at small strains are similar for different crosslinked particles. The macroscopic elastic deformation is mainly determined by the elastic properties of the polymer chain [30]; accordingly the effect of different crosslinking density is suppressed. However, as the strain increases over the level of $10 \%$, the effect of crosslinking becomes pronounced. The higher

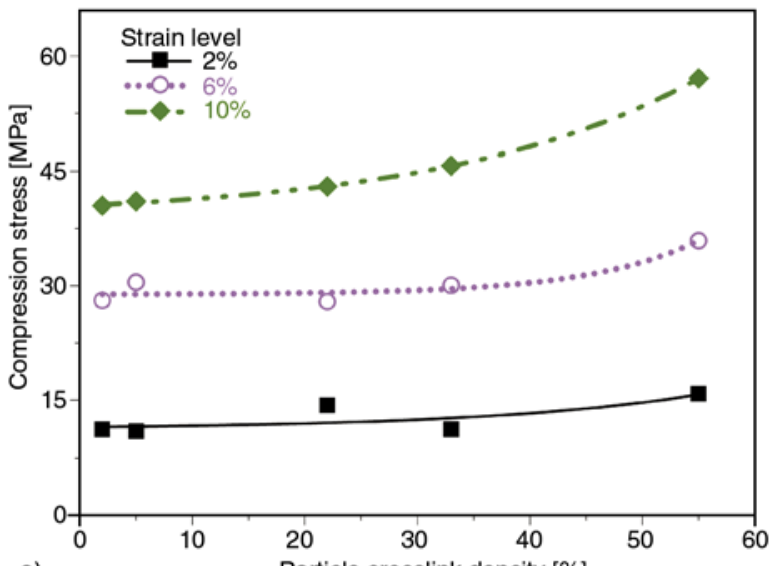

a)

Particle crosslink density [\%] crosslinking density induces stiffer behaviour, as shown in Figure $7 b$. At this stage, a significant rearrangement of the polymer chains for the slightly crosslinked particles is expected, whereas the structure is much more locked for the highly crosslinked ones. The major difference occurs at high strains where the two slightly crosslinked particles PSA and PSB can deform up to $80 \%$ without stress drop, while the highly crosslinked PSC, PSD and PSE show a complete fragmentation of the particles.

The compression stress of PS-DVB particles at different strain levels are summarized in Figure 8 where the distinct effect of crosslinking density is illustrated. At the strain level less than $10 \%$, the effect of crosslinking density is relatively mild, as shown in Figure 8a, for example the five groups of particles have nearly identical compression stress when the stain level is $2 \%$. As increasing the strain level above $10 \%$, the effect of the crosslinking density becomes more and more evident. To deform the

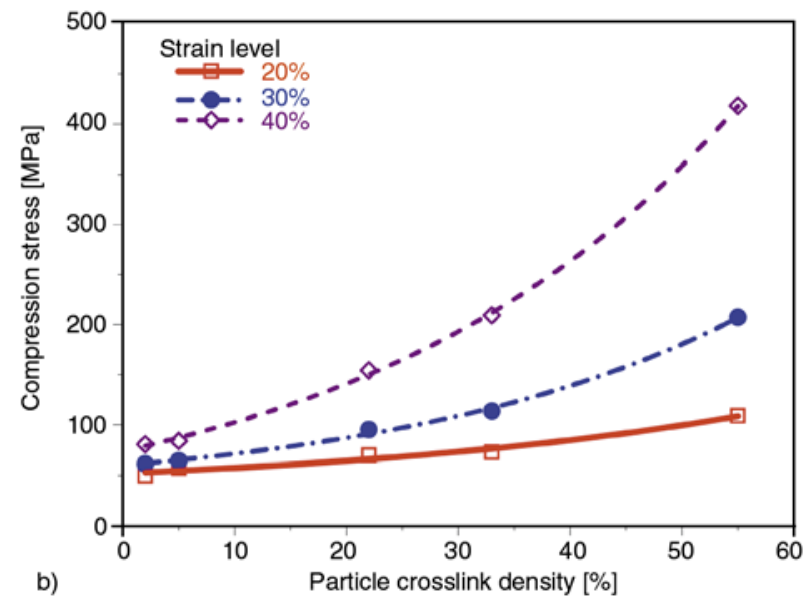

b)

Figure 8. The crosslinking density effect on the compression stress of five PS-DVB particles (a) at small strain scale and (b) at large strain scale. The lines are guides for the eye. 
five groups of particles to a relatively large strain level, the compression stress dramatically raise with the crosslinking density.

The deformation behaviour in polymer materials is often classified in three modes: plastic behaviour, rubber-like behaviour and brittle fracture behaviour [31]. Non-crosslinked linear polystyrene is found to exhibit brittle crazing in tension, while plastic behaviour in compression through shear banding or shear yielding [32]. Crazing is a localized yielding behaviour and characterized as whitening of the polymer in the region of maximum deformation through formation of micro-cracks, which are bridged by polymer fibrils. Shear banding, which is highly dependent on temperature and strain rate, is featured by planes of slip at $45^{\circ}$ to the direction of stress and involves the local orientation of the polymer. For bulky crosslinked polymers, it is known that the mechanical properties are sensitive to the crosslinking density [33]. Crosslinking increases the tightness of the polymer network and limits the molecular mobility of chains between the junctions. The randomly crosslinked network of polymers undergoes a transition from a fluid to an amorphous solid state behaviour as the crosslinking increases [34]. The characteristic length between crosslinking decreases with the increasing crosslinking density. This means that the available degrees of freedom in rearranging the molecular chain decreases with an increasing degree of crosslinking.

However, in the present work, the spherical geometry increases the complexities of the stress and strain distribution in the particle. The compression on the particles induces a drum-like pattern consisting of both compressive and tensile components. The maximum tensile stress induced by the compression is located in the circumferential direction Thus a number of crazes at the toroid surface and residual deformation are observed on the slightly crosslinked particles with plastic behaviour, whose deformation is comparable with the linear polystyrene. There is no definite yielding point on the stress-strain curves but a long plateau implying the plastic flow following by strain hardening, as shown in Figure $7 \mathrm{~b}$. With increasing crosslinking density, the crosslinking restricts the deformability and three highly crosslinked particles show viscoelastic behaviour. The SEM observation confirms full recovery of particles or fragmentation at high peak load with- out craze formation although there are compressive and tensile region during compression.

When increasing the crosslinking density from 5.0 to $21.3 \mathrm{wt} \%$, the particle deformation changes from plastic deformation to viscoelastic behaviour. Due to the lack of PS-DVB particles located in the region between 5.0 and $21.3 \mathrm{wt} \%$ of crosslinking density, the more detailed information of the shift of fracture pattern is not available. According to the nature of polymer materials, this change is influenced by strain rate and temperature. It is difficult to define an exact shift point of a critical crosslinking density.

It is worth noting that the deformation and fracture pattern of particles observed in this study is simply valid for the PS-DVB particles within the sampling crosslinking density. As mentioned above, the crosslinking density is determined by the amount of DVB containing two vinyl groups during particle preparation. When adding more DVB, it might be supersaturated and not fully reacted with styrene monomer. It will result in a lower effective crosslinking density than calculated from the amount of DVB.

\section{Conclusions}

By using a nanoindentation-based flat punch method, the effect of the crosslinking density on the mechanical properties of monodisperse PS-DVB particles has been systematically investigated. The monodisperse PS-DVB particles are prepared by the wellknown Ugelstad activated swelling method. The amount of crosslinking agent in the particles varies from 2.0 to $55.3 \%$ by weight and the diameter of particles is kept around $5 \mu \mathrm{m}$. The deformation mechanism of slightly crosslinked particles has been clarified to be plastic behaviour while highly crosslinking ones are viscoelastic. At the strain level below $10 \%$, particle deformation appears to be nearly independent of the crosslinking density. This is associated with the elasticity of styrene segments between neighboring crosslinking points. With the increase of strain, the crosslinking density effect becomes pronounced. The major difference occurs on the fracture behaviour. The slightly crosslinked particles show densely crazes and the failure of the highly crosslinked ones is presented by the direct evidence of fragmentation. The mechanism behind the observation is that the high 
crosslinking restricts the deformability of particles and also causes a heterogeneous microstructure.

\section{Acknowledgements}

This work is supported by The Research Council of Norway via NANOMAT KMB Projects NanoPCP (Grant No. NANOMAT-169737/S10) and MS2MP (Grant No. NANOMAT-187269), and long-term support from NorFab Norway. The authors gratefully acknowledge Wilhelm Dall for assistance with SEM operation.

\section{References}

[1] Ahmad H., Tauer K.: Effects of chain transfer agent on the radical polymerization of styrene in non-aqueous dispersion. Colloid and Polymer Science, 281, 686-689 (2003).

DOI: $10.1007 / \mathrm{s} 00396-002-0847-0$

[2] Toprak M. S., Mckenna B. J., Mikhaylova M., Waite J. H., Stucky G. D.: Spontaneous assembly of magnetic microspheres. Advanced Materials, 19, 1362-1368 (2007).

DOI: $10.1002 /$ adma.200602114

[3] Kim K., Kim Y., Ko N. R., Choe S.: Effect of molecular weight on the surface morphology of crosslinked polymer particles in the RITP-dispersion polymerization. Polymer, 52, 5439-5444 (2011).

DOI: $10.1016 /$ j.polymer.2011.10.015

[4] Kwon W-S., Paik K-W.: Experimental analysis of mechanical and electrical characteristics of metalcoated conductive spheres for anisotropic conductive adhesives (ACAs) interconnection. IEEE Transactions on Components and Packaging Technologies, 29, 528534 (2006).

DOI: $10.1109 /$ TCAPT.2006.880513

[5] Lin Y. C., Zhong J.: A review of the influencing factors on anisotropic conductive adhesives joining technology in electrical applications. Journal of Materials Science, 43, 3072-3093 (2008).

DOI: $10.1007 / \mathrm{s} 10853-007-2320-4$

[6] Kristiansen H., Liu J.: Overview of conductive adhesive interconnection technologies for LCDs. IEEE Transactions on Components, Packaging, and Manufacturing Technology, 21, 208-214 (1998). DOI: $10.1109 / 95.705466$

[7] Ellingsen T., Aune O., Ugelstad J., Hagen S.: Monosized stationary phases for chromatography. Journal of Chromatography A, 535, 147-161 (1990). DOI: $10.1016 / \mathrm{S} 0021-9673(01) 88940-8$

[8] Song J-S., Winnik M. A.: Monodisperse, micrometersized low molar mass polystyrene particles by twostage dispersion polymerization. Polymer, 47, 45574563 (2006).

DOI: $10.1016 /$ j.polymer.2006.04.025
[9] Tanaka T., Suzuki T., Saka Y., Zetterlund P. B., Okubo M.: Mechanical properties of cross-linked polymer particles prepared by nitroxide-mediated radical polymerization in aqueous micro-suspension. Polymer, 48, 3836-3843 (2007).

DOI: $10.1016 /$ j.polymer.2007.04.040

[10] Li L., Fang T.: Anisotropic conductive adhesive films for flip chip on flex packages. in 'Proceedings of the $4^{\text {th }}$ International Conference on Adhesive Joining and Coating Technology in Electronics Manufacturing, Espoo, Finland' 129-135 (2000).

DOI: 10.1109/ADHES.2000.860586

[11] Ahmed S. M.: Effects of agitation, and the nature of protective colloid on particle size during suspension polymerization. Journal of Dispersion Science and Technology, 5, 421-432 (1984). DOI: $10.1080 / 01932698408943229$

[12] Thomson B., Rudin A., Lajoie G.: Dispersion copolymerization of styrene and divinylbenzene. II. Effect of crosslinker on particle morphology. Journal of Applied Polymer Science, 59, 2009-2028 (1996).

DOI: 10.1002/(SICI)1097-4628(19960328)59:13<2009 $\because$ AID-APP6>3.0.CO;2-L

[13] Ishizu K., Tahara N.: Microsphere synthesis by emulsion copolymerization of methyl methacrylate with binary macromonomer blends. Polymer, 37, 1729-1734 (1996).

DOI: 10.1016/0032-3861(96)83726-8

[14] Okubo M., Shiozaki M., Tsujihiro M., Tsukuda Y.: Preparation of micron-size monodisperse polymer particles by seeded polymerization utilizing the dynamic monomer swelling method. Colloid and Polymer Science, 269, 222-226 (1991).

DOI: $10.1007 / \mathrm{BF} 00665495$

[15] Ugelstad J., Mørk P. C., Kaggerud K. H., Ellingsen T., Berge A.: Swelling of oligomer-polymer particles. New methods of preparation. Advances in Colloid and Interface Science, 13, 101-140 (1980). DOI: $10.1016 / 0001-8686(80) 87003-5$

[16] Ugelstad J., Berge A., Ellingsen T., Schmid R., Nilsen T. N., Mørk P. C., Stenstad P., Hornes E., Olsvik Ø.: Preparation and application of new monosized polymer particles. Progress in Polymer Science, 17, 87-161 (1992). DOI: 10.1016/0079-6700(92)90017-S

[17] Liu K-K.: Deformation behaviour of soft particles: A review. Journal of Physics D: Applied Physics, 39, R189-R199 (2006). DOI: $10.1088 / 0022-3727 / 39 / 11 / \mathrm{R} 01$

[18] Zhang Z. L., Kristiansen H., Liu J.: A method for determining elastic properties of micron-sized polymer particles by using flat punch test. Computational Materials Science, 39, 305-314 (2007).

DOI: $10.1016 /$ j.commatsci.2006.06.009 
[19] He J. Y., Zhang Z. L., Kristiansen H.: Nanomechanical characterization of single micron-sized polymer particles. Journal of Applied Polymer Science, 113, 13981405 (2009).

DOI: $10.1002 /$ app. 29913

[20] Kim D. O., Jin J. H.: Investigation for surface morphology and mechanical property variations of single polymer particles. Journal of Applied Polymer Science, 104, 2350-2360 (2007).

DOI: $10.1002 / a p p .25717$

[21] He J. Y., Zhang Z. L., Kristiansen H.: Compression properties of individual micron-sized acrylic particles. Materials Letters, 63, 1696-1698 (2009).

DOI: $10.1016 /$ j.matlet.2009.05.013

[22] He J. Y., Helland T., Zhang Z. L., Kristiansen H.: Fracture of micron-sized Ni/Au coated polymer particles. Journal of Physics D: Applied Physics, 42, 085405/1085405/5 (2009).

DOI: $10.1088 / 0022-3727 / 42 / 8 / 085405$

[23] He J. Y., Nagao S., Kristiansen H., Zhang Z. L.: Loading rate effects on the fracture of $\mathrm{Ni} / \mathrm{Au}$ nano-coated acrylic particles. Express Polymer Letters, 6, 198-203 (2012).

DOI: 10.3144/expresspolymlett.2012.22

[24] He J. Y., Zhang Z. L., Midttun M., Fonnum G., Modahl G. I., Kristiansen H., Redford K.: Size effect on mechanical properties of micron-sized PS-DVB polymer particles. Polymer, 49, 3993-3999 (2008). DOI: 10.1016/j.polymer.2008.07.015

[25] Ugelstad J.: Monodisperse polymer particles and dispersions thereof. U.S. Patent 4459378, USA (1984).

[26] Ding J., Xue G., Dai Q., Cheng R.: Glass transition temperature of polystyrene microparticles. Polymer, 34, 3325-3327 (1993). DOI: $\underline{10.1016 / 0032-3861(93) 90412-4}$
[27] Bandyopadhyay A., Valavala P. K., Clancy T. C., Wise K. E., Odegard G. M.: Molecular modeling of crosslinked epoxy polymers: The effect of crosslink density on thermomechanical properties. Polymer, 52, 2445-2452 (2011).

DOI: $10.1016 /$ j.polymer.2011.03.052

[28] Rey L., Duchet J., Galy J., Sautereau H., Vouagner D., Carrion L.: Structural heterogeneities and mechanical properties of vinyl/dimethacrylate networks synthesized by thermal free radical polymerisation. Polymer, 43, 4375-4384 (2002).

DOI: 10.1016/S0032-3861(02)00266-5

[29] VanLandingham M. R., Villarrubia J. S., Guthrie W. F., Meyers G. F.: Nanoindentation of polymers: An overview. Macromolecular Symposia, 167, 15-44 (2001). DOI: $10.1002 / 1521-3900(200103) 167: 1<15:: A I D-$ MASY15>3.0.CO;2-T

[30] Vakil U. M., Martin G. C.: Crosslinked epoxies: Network structure characterization and physical-mechanical properties. Journal of Applied Polymer Science, 46, 2089-2099 (1992). DOI: $10.1002 /$ app.1992.070461204

[31] Strobl G.: The physics of polymers. Springer, Heidelberg (2007).

[32] Chow T. S.: Stress-strain behavior of polymers in tension, compression, and shear. Journal of Rheology, 36, 1707-1717 (1992). DOI: $10.1122 / 1.550281$

[33] Wang S., Yaszemski M. J., Gruetzmacher J. A., Lu L.: Photo-crosslinked poly( $\varepsilon$-caprolactone fumarate) networks: Roles of crystallinity and crosslinking density in determining mechanical properties. Polymer, 49, 5692-5699 (2008).

DOI: $10.1016 /$ j.polymer.2008.10.021

[34] Ulrich S., Mao X., Goldbart P. M., Zippelius A.: Elasticity of highly cross-linked random networks. Europhysics Letters, 76, 677-682 (2006). DOI: $10.1209 / \mathrm{epl} / \mathrm{i} 2006-10310-7$ 